\section{(6) OPEN ACCESS}

\title{
Evaluation of the genetic overlap between osteoarthritis with body mass index and height using genome-wide association scan data
}

\author{
Katherine S Elliott, ${ }^{1 *}$ Kay Chapman, ${ }^{1,2, *}$ Aaron Day-Williams, ${ }^{3}$ Kalliope Panoutsopoulou, ${ }^{3}$ \\ Lorraine Southam, ${ }^{1,3}$ Cecilia M Lindgren, ${ }^{1}$ the GIANT consortium ${ }^{4 *}$ Nigel Arden, ${ }^{5,6}$ \\ Nadim Aslam, ${ }^{7}$ Fraser Birrell, ${ }^{8,9}$ lan Carluke, ${ }^{9}$ Andrew Carr ${ }^{2,4}$ Panos Deloukas, ${ }^{3}$ \\ Michael Doherty, ${ }^{10}$ John Loughlin, ${ }^{11}$ Andrew McCaskie, ${ }^{11,12}$ William E R Ollier, ${ }^{13}$ \\ Ashok Rai, ${ }^{14}$ Stuart Ralston, ${ }^{15}$ Mike R Reed ${ }^{9}$ Timothy D Spector, ${ }^{16}$ Ana M Valdes, ${ }^{16}$ \\ Gillian A Wallis, ${ }^{17}$ Mark Wilkinson, ${ }^{18}$ the arcOGEN consortium, ${ }^{4}$ Eleftheria Zeggini ${ }^{3}$
}

\begin{abstract}
- Additional supplementary materials are published online only. To view these files please visit the journal online (http://dx.doi.org/10.1136/ annrheumdis-2012-202081).
\end{abstract}

For numbered affiliations see end of article.

\section{Correspondence to} Eleftheria Zeggini, Wellcome Trust Sanger Institute, Hinxton, Cambridge, CB10 1SA, UK; eleftheria@sanger.ac.uk

*For GIANT and arcOGEN consortium members see supplementary material (available online only).

Received 28 May 2011 Accepted 19 July 2012 Published Online First 6 September 2012

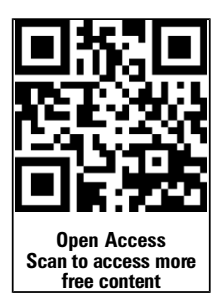

\section{ABSTRACT}

Objectives Obesity as measured by body mass index (BMI) is one of the major risk factors for osteoarthritis. In addition, genetic overlap has been reported between osteoarthritis and normal adult height variation. We investigated whether this relationship is due to a shared genetic aetiology on a genome-wide scale.

Methods We compared genetic association summary statistics (effect size, $p$ value) for BMl and height from the GIANT consortium genome-wide association study (GWAS) with genetic association summary statistics from the arcOGEN consortium osteoarthritis GWAS.

Significance was evaluated by permutation. Replication of osteoarthritis association of the highlighted signals was investigated in an independent dataset. Phenotypic information of height and BMI was accounted for in a separate analysis using osteoarthritis-free controls. Results We found significant overlap between osteoarthritis and height $\left(p=3.3 \times 10^{-5}\right.$ for signals with $p \leq 0.05$ ) when the GIANT and arc0GEN GWAS were compared. For signals with $p \leq 0.001$ we found 17 shared signals between osteoarthritis and height and four between osteoarthritis and BMI. However, only one of the height or BMI signals that had shown evidence of association with osteoarthritis in the arcOGEN GWAS was also associated with osteoarthritis in the independent dataset: rs12149832, within the FTO gene (combined $p=2.3 \times 10^{-5}$ ). As expected, this signal was attenuated when we adjusted for BMI.

Conclusions We found a significant excess of shared signals between both osteoarthritis and height and osteoarthritis and BMI, suggestive of a common genetic aetiology. However, only one signal showed association with osteoarthritis when followed up in a new dataset.

\section{INTRODUCTION}

Osteoarthritis is a common complex disease of synovial joints characterised by degeneration of hyaline cartilage and bone remodelling, usually affecting middle-aged to elderly individuals. It is a leading cause of pain and chronic disability worldwide. ${ }^{1}$ Comorbidities such as obesity are frequently observed with osteoarthritis and epidemiological studies have noted a link between osteoarthritis and obesity as measured by body mass index (BMI). In particular, reports show a consistent relationship between overweight measures and knee osteoarthritis. ${ }^{2}$ Some population studies have demonstrated that the weight of individuals at age 37 years (median) could predict the onset of knee osteoarthritis 36 years later. ${ }^{1}$ In addition, a decrease in BMI of two units over the 10 years preceding diagnosis can reduce the odds of knee osteoarthritis. ${ }^{3}$ A Norwegian population-based study of approximately 265000 individuals concluded that the risk of developing hip osteoarthritis was dependent on the age at which weight gain was most dramatic. Younger adults ( $<20$ years) are at greater risk compared with older individuals (>30 years). ${ }^{4}$ In a large prospective populationbased cohort from Iceland the incidence of clinically severe osteoarthritis (as indicated by arthroplasty), in relation to measures of overweight, found that $36 \%$ and $50 \%$ of those with hip and knee osteoarthritis, respectively, had a BMI greater than $30 .^{5}$ This is compared with a national prevalence of $17 \%$ of the adult population (the International Obesity Task force, http://www.iotf. org/). Furthermore, the Chingford Study has demonstrated that in middle-aged women a one unit increase in BMI is associated with a $10 \%$ increased risk of total knee replacement in the following 19 years. ${ }^{6}$ There have been a number of large-scale genome-wide association studies (GWAS) for obesity and/or BMI, establishing several genetic loci at genome-wide significance association levels. ${ }^{7-13}$ Based on the well-established epidemiological link, we hypothesise here that osteoarthritis and obesity may have a shared genetic background. ${ }^{14}$

Variation in human adult height is also highly heritable. Numerous studies have identified over 180 loci to be associated with the trait. ${ }^{15-18}$ There is weak or conflicting evidence for shared genetic determinants between osteoarthritis and height. However, for example, the functional single nucleotide polymorphism (SNP) (rs143383, T/C) in the $5^{\prime}$ untranslated region of the GDF5 gene, previously observed to be significantly associated 
with osteoarthritis in Asian and European cohorts, is also significantly associated with normal variation in human height. ${ }^{19-}$

${ }^{23}$ In addition, height itself has been reported to be a risk factor for non-generalised severe hip osteoarthritis even after adjusting for age, gender and $\mathrm{BMI}^{24}$

The aim of this study was to carry out an investigation of the genetic overlap between osteoarthritis and the two traits of BMI and height by examining the overlap of SNPs association signals across the genome. This may uncover possible common mechanistic pathways.

\section{MATERIALS AND METHODS}

\section{Description of datasets}

Genome-wide summary statistics (effect size, $p$ values) for BMI and height from the Genetic Investigation of Anthropometric Traits (GIANT) consortium GWAS were compared with genome-wide osteoarthritis data from the arcOGEN consortium. The GIANT consortium has brought together GWAS data from 46 studies. $^{25} 26$ Overlap analysis with osteoarthritis utilised 2400344 SNPs and 32387 individuals from the BMI dataset and 2834208 SNPs and 133653 individuals from the height dataset.

The arcOGEN GWAS was carried out in two stages and includes a total of 7567 osteoarthritis cases from the UK (ascertained by radiographic evidence of disease, Kellgren-Lawrence score $\geq 2$, or clinical evidence of the disease to a level requiring total joint replacement) genotyped on the Illumina HumanHap 610-Quad panel. Stage 1 of the arcOGEN GWAS was employed in the main overlap analysis and included 3177 osteoarthritis cases and 4894 population-based controls from the UK (WTCCC2). ${ }^{27}$ Genotypes of 17 SNPs that were imputed in arcOGEN stage 1 were validated by direct typing using Sequenom in the stage 1 cases $(n=2949)$ and examining concordance. Replication of association with osteoarthritis for the signals highlighted from the overlap analysis (tables 1 and 2) was carried out using 4324 stage 2 cases from the arcOGEN Consortium and 6518 population-based controls (from the WTCCC2, T1DGC, ALSPAC study and PoBI studies) (see supplementary methods, available online only). SNPs that were not genotyped in the stage 2 arcOGEN GWAS or did not pass quality control were genotyped with Sequenom in 5165 cases and 6115 controls (see supplementary methods, available online only).

Analysis accounting for phenotypic information of height and BMI was carried out using 1671 unrelated female individuals from the osteoarthritis-free TwinsUK cohort as a control set. This cohort is ascertained to study the heritability of age-related diseases and contains full phenotypic information for osteoarthritis status as well as height and BMI. ${ }^{24}$ Additional quality control that was performed for this study is described in the supplementary methods (available online only). arcOGEN stage 1 female cases $(n=1009$ for height; $n=1358$ for BMI) were utilised for these analyses.

\section{Osteoarthritis replication genotyping}

Osteoarthritis association signals at directly typed variants, highlighted in tables 3 and 4, were followed up through in-silico replication using stage 2 arcOGEN GWAS data (see supplementary methods, available online only). Association signals at imputed variants were followed up by carrying out de-novo genotyping in 5165 arcOGEN stage 2 cases and 6115 WTCCC2 controls using the Sequenom MassArray iPLex Gold assay at the Wellcome Trust Sanger Institute. Genotypes were assigned using the MassArray TyperAnalyser software V.4.0 (Sequenom). All genotypes were confirmed manually and passed standard quality control checks (see supplementary methods, available online only).

\section{Analysis strategy}

We carried out pairwise comparisons between osteoarthritis and height and between osteoarthritis and BMI genome-wide summary statistics. For each comparison, we focused on the intersection of SNPs for which summary statistics were present in both GWAS. We then sorted these SNPs based on association $p$ value for osteoarthritis. This list of SNPs was then thinned to an independent unlinked set using an $\mathrm{r}^{2}$ threshold of 0.05 based on HapMap CEU release \#27. Starting with the first SNP in the list, any subsequent SNP with $\mathrm{r}^{2}>0.05$ was removed and then the next available SNP was taken. This

Table 1 Shared genetic determinants $\left(p \leq 1.0 \times 10^{-3}\right)$ between osteoarthritis and height

\begin{tabular}{|c|c|c|c|c|c|c|c|c|c|}
\hline SNP & Chromosome & Allele & Osteoarthritis $p$ value & Osteoarthritis OR & $95 \% \mathrm{Cl}$ & Height p value & Height OR & $95 \% \mathrm{CI}$ & Nearest gene \\
\hline rs2744718 & 1 & $\mathrm{~T}$ & $8.9 \times 10^{-5}$ & 1.20 & 1.10 to 1.31 & $3.5 \times 10^{-5}$ & 0.98 & 0.97 to 0.99 & WNT4 \\
\hline rs6670486 & 1 & $\mathrm{~T}$ & $9.6 \times 10^{-5}$ & 1.16 & 1.07 to 1.24 & $6.5 \times 10^{-7}$ & 0.98 & 0.98 to 0.98 & COL11A1 \\
\hline rs4833772 & 4 & $G$ & $2.5 \times 10^{-4}$ & 1.12 & 1.05 to 1.19 & $1.3 \times 10^{-5}$ & 1.02 & 1.01 to 1.02 & TMEM155 \\
\hline rs572004 & 6 & G & $2.4 \times 10^{-4}$ & 1.14 & 1.06 to 1.22 & $7.5 \times 10^{-5}$ & 0.98 & 0.97 to 0.99 & EYA4 \\
\hline rs3822856 & 6 & $A$ & $9.2 \times 10^{-4}$ & 1.12 & 1.05 to 1.19 & $6.5 \times 10^{-4}$ & 0.99 & 0.98 to 0.99 & NT5DC1 \\
\hline rs1635853 & 7 & $\mathrm{~T}$ & $2.0 \times 10^{-5}$ & 1.15 & 1.08 to 1.22 & $8.8 \times 10^{-21}$ & 1.04 & 1.03 to 1.04 & JAZF1 \\
\hline rs10094727 & 8 & A & $3.7 \times 10^{-4}$ & 1.23 & 1.10 to 1.39 & $6.0 \times 10^{-5}$ & 0.97 & 0.06 to 0.98 & MSR1 \\
\hline rs9657371 & 8 & $A$ & $1.3 \times 10^{-4}$ & 1.13 & 1.06 to 1.21 & $2.7 \times 10^{-5}$ & 0.98 & 0.97 to 0.99 & CSMD1 \\
\hline rs11991139 & 8 & C & $6.2 \times 10^{-5}$ & 1.14 & 1.06 to 1.22 & $4.3 \times 10^{-4}$ & 0.98 & 0.98 to 0.99 & $B L K$ \\
\hline rs3808880 & 9 & G & $7.5 \times 10^{-4}$ & 1.13 & 1.05 to 1.21 & $6.8 \times 10^{-4}$ & 1.01 & 1.01 to 1.02 & ROD1 \\
\hline rs11198893 & 10 & A & $3.8 \times 10^{-4}$ & 1.22 & 1.09 to 1.36 & $5.7 \times 10^{-4}$ & 1.02 & 1.01 to 1.03 & GRK5 \\
\hline rs7932272 & 11 & $A$ & $1.1 \times 10^{-4}$ & 1.30 & 1.12 to 1.52 & $2.4 \times 10^{-7}$ & 1.04 & 1.03 to 1.06 & PACS1 \\
\hline rs7297051 & 12 & $\mathrm{~T}$ & $3.3 \times 10^{-4}$ & 1.14 & 1.05 to 1.23 & $6.0 \times 10^{-5}$ & 1.02 & 1.01 to 1.03 & PTHLH \\
\hline rs 10506474 & 12 & C & $5.2 \times 10^{-4}$ & 1.15 & 1.06 to 1.27 & $4.4 \times 10^{-5}$ & 0.99 & 0.97 to 0.99 & HMGA2 \\
\hline rs4793927 & 17 & C & $1.4 \times 10^{-4}$ & 1.14 & 1.06 to 1.22 & $3.0 \times 10^{-6}$ & 1.02 & 1.01 to 1.03 & HOХВ3 \\
\hline rs2864419 & 19 & $\mathrm{G}$ & $8.4 \times 10^{-5}$ & 1.14 & 1.07 to 1.21 & $1.1 \times 10^{-12}$ & 1.03 & 1.02 to 1.04 & DOT1L \\
\hline rs8105885 & 19 & $\mathrm{~T}$ & $7.9 \times 10^{-4}$ & 1.20 & 1.08 to 1.37 & $4.3 \times 10^{-4}$ & 0.98 & 0.96 to 0.98 & ZNF98 \\
\hline
\end{tabular}

SNP, single nucleotide polymorphism. 
Table 2 Shared genetic determinants $\left(p \leq 1.0 \times 10^{-3}\right)$ between osteoarthritis and BMI

\begin{tabular}{lllllllll}
\hline SNP & Chromosome & Allele & Osteoarthritis p value & 0steoarthritis OR & 95\% CI & BMI p value & BMI z score & Nearest gene \\
\hline rs4856346 & 3 & T & $6.1 \times 10^{-4}$ & 1.14 & 1.05 to 1.22 & $7.1 \times 10^{-4}$ & 3.48 & GBE1 \\
rs7828042 & 8 & G & $1.1 \times 10^{-4}$ & 1.14 & 1.07 to 1.22 & $2.1 \times 10^{-4}$ & 4.05 & SLURP1 \\
rs7203219 & 16 & T & $3.5 \times 10^{-4}$ & 1.19 & 1.08 to 1.31 & $3.4 \times 10^{-4}$ & 3.87 & GPR139 \\
rs12149832 & 16 & A & $2.8 \times 10^{-4}$ & 1.12 & 1.06 to 1.20 & $1.9 \times 10^{-16}$ & 8.47 & FTO \\
\hline
\end{tabular}

BMI, body mass index; SNP, single nucleotide polymorphism.

continued until a set of independent SNPs was obtained (osteoarthritis-BMI $n=62$ 280, osteoarthritis-height $n=64702$ ).

We investigated the distribution of $\mathrm{p}$ values above and below given thresholds $(0.5,0.1,0.05,0.04,0.03,0.02,0.01,0.005$, $0.001,0.0005)$ for each trait. The distribution of counts in the resulting $2 \times 2$ contingency tables was analysed using the $\chi^{2}$ test. A significant excess of signals with $p$ values less than the given threshold for both phenotypes was taken to indicate a concurrence of signals.

In addition, we examined the SNPs for each comparative analysis of osteoarthritis-height and osteoarthritis-BMI to see if there was an overabundance of discordant or concordant risk alleles between the datasets (see supplementary methods, available online only).

\section{Permutations}

Based on the results obtained for the analysis of the signal overlap between the osteoarthritis-height and osteoarthritisBMI comparisons (table 5), we selected $p$ value thresholds of 0.001 (osteoarthritis-BMI) and 0.05 (osteoarthritis-height) for further follow-up. We permuted the $\mathrm{p}$ value signals for the entire datasets as well as for linkage disequilibrium (LD)-thinned data of $\mathrm{r}^{2}=0.2$ and 0.05 . We generated 100000 permutations of the arcOGEN case-control data using PLINK ${ }^{28}$ (make-perm-pheno command) and performed a GWAS for each permutation under the log-additive model. We carried out the overlap analysis for each permuted case-control dataset considering only SNPs that were directly typed in arcOGEN and present in the GIANT data. We thus generated a null distribution of $p$ values. From this we calculated the probability of seeing an overlap $p$ value equal to or less than the original $p$ value for directly typed SNPs.

In addition, we sought to get a more precise empirical $p$ value for the osteoarthritis-height comparison as this gave the most compelling results for the signal overlap analysis (table 5). Using the LD-thinned data $\left(r^{2}=0.05\right)$ and $p$ value threshold of 0.05 , we generated 500000000 permutations of the arcOGEN and GIANT height datasets by permuting which $\mathrm{p}$ value was associated with which SNP. We performed 500000000 overlap analyses by randomly choosing without replacement a permutation from each dataset to generate the null distribution of overlap $p$ values, given a specific distribution of original $p$ values. From this constructed null distribution of $p$ values we calculated the probability of seeing an overlap $p$ value equal to or less than the original overlap $p$ value for the entire dataset.

\section{Replication of osteoarthritis association for overlapping signals}

Case-control association analysis under the log-additive model was carried out using PLINK for directly typed SNPs and SNPTEST for imputed SNPs. ${ }^{28} 30$ Combined estimates of OR and $\mathrm{p}$ values for stages 1 and 2 of arcOGEN were obtained

Table 3 Replication of osteoarthritis association at shared genetic determinants between osteoarthritis and height

\begin{tabular}{|c|c|c|c|c|c|c|c|c|c|c|}
\hline \multirow[b]{2}{*}{ SNP } & \multirow[b]{2}{*}{ Chromosome } & \multicolumn{3}{|l|}{ Stage 1} & \multicolumn{3}{|l|}{ Stage 2} & \multicolumn{3}{|l|}{ Combined } \\
\hline & & p Value & OR & $95 \% \mathrm{Cl}$ & P Value & OR & $95 \% \mathrm{CI}$ & p Value & OR & $95 \% \mathrm{Cl}$ \\
\hline rs2744718†‡ & 1 & $8.9 \times 10^{-5}$ & 1.20 & 1.10 to 1.31 & Failed $\mathrm{QC}$ & Failed OC & & & & \\
\hline rs4833772* & 4 & $2.5 \times 10^{-4}$ & 1.12 & 1.05 to 1.19 & 0.24 & 0.97 & 0.92 to 1.02 & $1.6 \times 10^{-1}$ & 1.03 & 0.99 to 1.07 \\
\hline rs572004 & 6 & $2.4 \times 10^{-4}$ & 1.14 & 1.06 to 1.22 & 0.29 & 1.03 & 0.97 to 1.09 & $9.0 \times 10^{-4}$ & 1.07 & 1.03 to 1.14 \\
\hline rs3822856 & 6 & $9.2 \times 10^{-4}$ & 1.12 & 1.05 to 1.19 & 0.29 & 1.02 & 0.97 to 1.09 & $2.5 \times 10^{-3}$ & 1.06 & 1.02 to 1.11 \\
\hline rs9657371‡ & 8 & $1.3 \times 10^{-4}$ & 1.13 & 1.06 to 1.21 & 0.60 & 1.02 & 0.96 to 1.07 & $3.7 \times 10^{-3}$ & 1.06 & 1.02 to 1.11 \\
\hline rs11991139* & 8 & $6.2 \times 10^{-5}$ & 1.14 & 1.06 to 1.22 & 0.37 & 1.02 & 1.0 to 1.09 & $3.1 \times 10^{-3}$ & 1.05 & 1.02 to 1.10 \\
\hline rs $3808880^{*}$ & 9 & $7.5 \times 10^{-4}$ & 1.13 & 1.05 to 1.21 & 0.03 & 0.94 & 0.88 to 0.99 & $5.6 \times 10^{-1}$ & 1.01 & 0.97 to 1.06 \\
\hline rs11198893†ł & 10 & $3.8 \times 10^{-4}$ & 1.22 & 1.09 to 1.36 & Failed OC & & & & & \\
\hline rs7932272 & 11 & $1.1 \times 10^{-4}$ & 1.30 & 1.12 to 1.52 & 0.08 & 1.10 & 0.99 to 1.20 & $5.2 \times 10^{-4}$ & 1.16 & 1.06 to 1.26 \\
\hline rs8105885 & 19 & $7.9 \times 10^{-4}$ & 1.20 & 1.08 to 1.37 & 0.15 & 0.93 & 0.85 to 1.03 & $3.2 \times 10^{-1}$ & 1.04 & 0.96 to 1.12 \\
\hline
\end{tabular}

*Proxies used for analysis due to failure of SNP in stage 2 replication. Proxy for rs4833772 is rs $4833233\left(r^{2}=1\right)$, for rs 11991139 is rs $13280813\left(r^{2}=0.94\right)$ and for $r s 3808880$ is rs13293285 $\left(r^{2}=0.89\right)$.

tNo proxies found for $r^{2}>0.3$ (rs2744718); $r^{2}>0.43$ (rs11198893).

‡Directly typed SNP analysed from arcOGEN genome-wide association scan.

$\mathrm{QC}$, qualtiy control; SNP, single nucleotide polymorphism. 
Table 4 Replication of osteoarthritis association at shared genetic determinants between osteoarthritis and body mass index

\begin{tabular}{|c|c|c|c|c|c|c|c|c|c|c|}
\hline \multirow[b]{2}{*}{ SNP } & \multirow[b]{2}{*}{ Chromosome } & \multicolumn{3}{|l|}{ Stage 1} & \multicolumn{3}{|l|}{ Stage 2} & \multicolumn{3}{|l|}{ Combined } \\
\hline & & p Value & OR & $95 \% \mathrm{Cl}$ & p Value & $\mathbf{O R}$ & $95 \% \mathrm{Cl}$ & p Value & OR & $95 \% \mathrm{CI}$ \\
\hline rs $4856346^{*}$ & 3 & $6.1 \times 10^{-4}$ & 1.14 & 1.06 to 1.23 & 0.87 & 0.99 & 0.94 to 1.06 & 0.041 & 1.05 & 1.00 to 1.10 \\
\hline rs7203219† & 16 & $3.5 \times 10^{-4}$ & 1.19 & 1.08 to 1.31 & 0.92 & 1.01 & 0.93 to 1.09 & 0.016 & 1.08 & 1.01 to 1.15 \\
\hline rs 12149832* & 16 & $2.8 \times 10^{-4}$ & 1.12 & 1.06 to 1.20 & 0.009 & 1.07 & 1.02 to 1.14 & $2.3 \times 10^{-5}$ & 1.10 & 1.05 to 1.15 \\
\hline
\end{tabular}

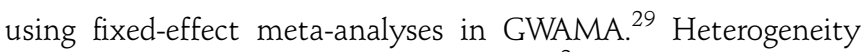
was checked using the Cochran's $\mathrm{Q}$ and $\mathrm{I}^{2}$ statistics.

\section{Analysis with adjustment for BMI and height}

In order to adjust for height and BMI as covariates, we repeated the case-control analysis for stage 1 of the arcOGEN dataset using the TwinsUK cohort as controls. Analysis was carried out using PLINK when the SNPs were directly typed or SNPTEST ${ }^{30}$ when they were imputed. The analysis was performed twice; with and without an adjustment for height and BMI.

\section{RESULTS}

\section{Overlapping signals and permutations}

Our findings suggest an excess of shared signals both between osteoarthritis and height and osteoarthritis and BMI. A comparison of signals indicates an excess of sharing at $p$ value thresholds of $0.05,0.04,0.03,0.02$ and 0.01 for osteoarthritis and height; there is evidence for overlap between osteoarthritis and BMI at the $\mathrm{p}$ value thresholds of $0.005,0.001$ and 0.0005 (table 5).

To test the strength of the observed overlap we ran a series of permutations (table 6). The LD-thinned datasets provide the most robust results because the probability of seeing an overlap $p$ value equal to or less than the original analysis $p$ value by chance is unlikely. Both comparisons of osteoarthritis-height and osteoarthritis-BMI showed an excess of overlapping signals $\left(p=1.4 \times 10^{-3}\right.$ for the $0.05 p$ value threshold and $p=2.28 \times 10^{-2}$ for the $0.001 \mathrm{p}$ value threshold). Based on these results we performed 500000000 permutations of the entire LD-thinned

Table 5 Analysis of shared excess signals between osteoarthritis and normal height variation and osteoarthritis and BMI

\begin{tabular}{|c|c|c|c|c|}
\hline \multirow{3}{*}{$\begin{array}{l}\text { Total no of } \\
\text { SNPs } \\
\text { Signal } \\
\text { definition } \\
\text { (p value) }\end{array}$} & \multicolumn{2}{|c|}{$\begin{array}{l}\text { Osteoarthritis-normal adult } \\
\text { height comparison }\end{array}$} & \multicolumn{2}{|c|}{$\begin{array}{l}\text { Osteoarthritis-BMI } \\
\text { comparison }\end{array}$} \\
\hline & \multicolumn{2}{|l|}{62280} & \multicolumn{2}{|l|}{64702} \\
\hline & $\begin{array}{l}\text { Overlapping } \\
\text { SNPs (n) }\end{array}$ & $\begin{array}{l}\text { p Value for } \\
\text { overlap }\end{array}$ & $\begin{array}{l}\text { Overlapping } \\
\text { SNPs (n) }\end{array}$ & $\begin{array}{l}\text { p Value for } \\
\text { overlap }\end{array}$ \\
\hline 0.5 & 28194 & 0.5189 & 23526 & 0.4299 \\
\hline 0.1 & 3571 & 0.0026 & 1867 & 0.5814 \\
\hline 0.05 & 1491 & $3.04 \times 10^{-5}$ & 584 & 0.7483 \\
\hline 0.04 & 1160 & $1.00 \times 10^{-6}$ & 412 & 0.6315 \\
\hline 0.03 & 814 & $1.00 \times 10^{-6}$ & 267 & 0.1715 \\
\hline 0.02 & 511 & $1.00 \times 10^{-16}$ & 143 & 0.0368 \\
\hline 0.01 & 213 & $6.20 \times 10^{-4}$ & 50 & 0.0356 \\
\hline 0.005 & 92 & 0.0173 & 20 & 0.0057 \\
\hline 0.001 & 17 & 0.5999 & 4 & $1.2 \times 10^{-5}$ \\
\hline 0.0005 & 8 & 0.1278 & 3 & $4.8 \times 10^{-17}$ \\
\hline
\end{tabular}

BMI, body mass index; SNP, single nucleotide polymorphism. $\left(\mathrm{r}^{2}=0.05\right)$ height dataset. This showed that the probability of seeing a $p$ value less than or equal to $3.04 \times 10^{-5}$ was $3.3 \times 10^{-5}$. A total of 17 SNPs with $p$ values of $1.0 \times 10^{-3}$ or less were shared between osteoarthritis and height (table 1), while four SNPs were shared between osteoarthritis and BMI (table 2). These SNPs were distributed throughout the genome 11 chromosomes in the osteoarthritis-height comparison and three chromosomes in the osteoarthritis-BMI comparison). Some of these signals, such as the ones near COL11A1, PTHLH and FTO are well-known loci with established associations with bone development, bone mineral density and obesity, respectively. 81931

\section{Replication of osteoarthritis association}

To evaluate the observed overlap further we attempted to replicate osteoarthritis association of these 21 signals employing stage 2 of the arcOGEN dataset (tables 3 and 4). Seven of the SNP failed quality control in stage 2 and proxies $\left(r^{2}>0.85\right)$ were sought. No proxies were found for two of the seven SNPs, rs2744718 and rs11198893. Of the 19 SNPs successfully taken forward for validation, rs12149832 on chromosome 16 within the FTO gene was the only one found to be associated $(p<0.01)$ with osteoarthritis in the replication dataset $(p=0.009$, in the same direction). The combined $p$ value of both stages increased in significance for this SNP relative to stage 1 alone $\left(p=2.8 \times 10^{-4}\right.$ for stage 1 vs $p=2.3 \times 10^{-5}$ for stages 1 and 2 combined, table 4 ).

\section{Adjustment for BMI and height}

Adjustment for height and BMI (tables 7 and 8) only affected the signal at the FTO SNP rs12149832. Here we found an eightfold increase in the $p$ value after adjustment for BMI $(p=0.22576)$ compared with the unadjusted result $(p=0.029219)$.

\section{DISCUSSION}

Identification of the genetic loci contributing to variation in quantitative traits such as height and BMI, and risk of osteoarthritis could help elucidate possible mechanistic pathways. There is an established genetic link between height and osteoarthritis. The pleiotropic action of GDF5 on human height is an example that may shed light on shared signalling functions and pathways affecting the two traits. ${ }^{19}$ Epidemiological evidence has also suggested a link between osteoarthritis and BMI. $^{32}$ It is plausible that these traits also share genetic associations and we carried out a SNP-by-SNP pairwise comparison of GWAS data to investigate their genetic overlap.

We obtained evidence for overlap of association signals between osteoarthritis and height and between osteoarthritis and BMI at different definition thresholds, corroborated by permutation analyses to obtain empirical $p$ values. We investigated specific signals 
Table 6 Permutation results for osteoarthritis-height ( $p$ value threshold 0.05 ) and osteoarthritis-BMl overlap ( $p$ value threshold 0.001 )

\begin{tabular}{|c|c|c|c|c|c|c|}
\hline & \multicolumn{3}{|c|}{ Osteoarthritis-adult height comparison } & \multicolumn{3}{|c|}{ Osteoarthritis-BMI comparison } \\
\hline & All data & $r^{2}=0.2$ & $r^{2}=0.05$ & All data & $r^{2}=0.2$ & $r^{2}=0.05$ \\
\hline No of SNPs $†$ & 489098 & 63802 & 27728 & 461707 & 55275 & 24599 \\
\hline Tested $p$ value ${ }^{* *}$ & $3.30 \times 10^{-6}$ & $5.33 \times 10^{-6}$ & $1.10 \times 10^{-3}$ & $6.19 \times 10^{-11}$ & $2.91 \times 10^{-4}$ & $2.25 \times 10^{-4}$ \\
\hline No of permutations & 100000 & 100000 & 100000 & 100000 & 100000 & 100000 \\
\hline Permutation $\mathrm{p}$ value & 0.0128 & $<1 \times 10^{-5}$ & 0.0014 & 0.00374 & 0.04716 & 0.02288 \\
\hline
\end{tabular}

tDirectly typed only analysed.

*p Value of original analysis.

**p Value of the overlap for directly typed SNPs only. This is the $\mathrm{p}$ value that the permutation analysis is tested against.

$\mathrm{BMI}$, body mass index; SNPs, single nucleotide polymorphism.

that may be representative of these findings and looked at all SNPs with $p \leq 1.0 \times 10^{-3}$ for both comparisons. Some signals reside in the vicinity of genes, such as the structural protein collagen gene COL11A1 and the parathyroid hormone-related protein PTHLH that regulates endochondral bone development, which have previously been identified as possible candidates for osteoarthritis susceptibility. ${ }^{33-36}$ For the osteoarthritis-BMI comparison the FTO gene for obesity was highlighted.

Using a second dataset we attempted to replicate the osteoarthritis association of overlapping signals. The fact that the FTO locus was the only one to replicate in our second osteoarthritis dataset suggests that the other signals may have been false positive signals for osteoarthritis, or low power in the replication cohort. Adjustment for BMI attenuated this osteoarthritis signal, indicating that the primary association is with BMI.

The established osteoarthritis and height overlapping signal rs143383 located in GDF5 was not identified in this analysis. We found it to be strongly associated with height $\left(p=1.94 \times 10^{-50}\right)$, but not associated with osteoarthritis in the arcOGEN dataset $(p=0.602)$. Although association between the GDF5 locus and hip and knee osteoarthritis was first reported in a study of Japanese and Chinese individuals in $2007,{ }^{20}$ it took several years and large-scale meta-analysis efforts to replicate the association robustly in European populations. ${ }^{21-23}$ In addition to allele frequency disparities between ethnic groups, this observation also highlights the limited power $\left(<10 \%\right.$ at $\left.\alpha=5 \times 10^{-8}\right)$ of a dataset such as arCOGEN (comprising 3177 cases and 4894 controls) to detect a signal with modest effect (OR 1.15) and common risk allele frequency $\left(\sim 0.60\right.$ for the GDF5 signal). ${ }^{27}$ Our results should be interpreted within the power constraints of our study. First, osteoarthritis is a heterogeneous disease and the definition of the cases here was primarily based on painful rather than structural osteoarthritis. Second, we examined GWAS platform SNP content rather than known causal variants. Finally, the osteoarthritis GWAS used population-based controls, which can dilute power due to misclassifications of cases as controls in a common disease such as osteoarthritis.

In conclusion, our genome-wide comparison of GIANT and arcOGEN generated evidence for an overall excess of overlapping signals between osteoarthritis and the two quantitative traits of BMI and height. The FTO signal was robustly associated with BMI and osteoarthritis, and showed evidence of association in the replication osteoarthritis dataset. This signal underpins the known epidemiological link between BMI and osteoarthritis, and represents the single largest genetic effect for BMI, which may have facilitated its identification as a shared locus. Better-powered GWAS datasets, along with large-scale replication samples, will help unveil additional shared loci and highlight common biological pathways.

Table 7 Results of osteoarthritis association analysis adjusting for height

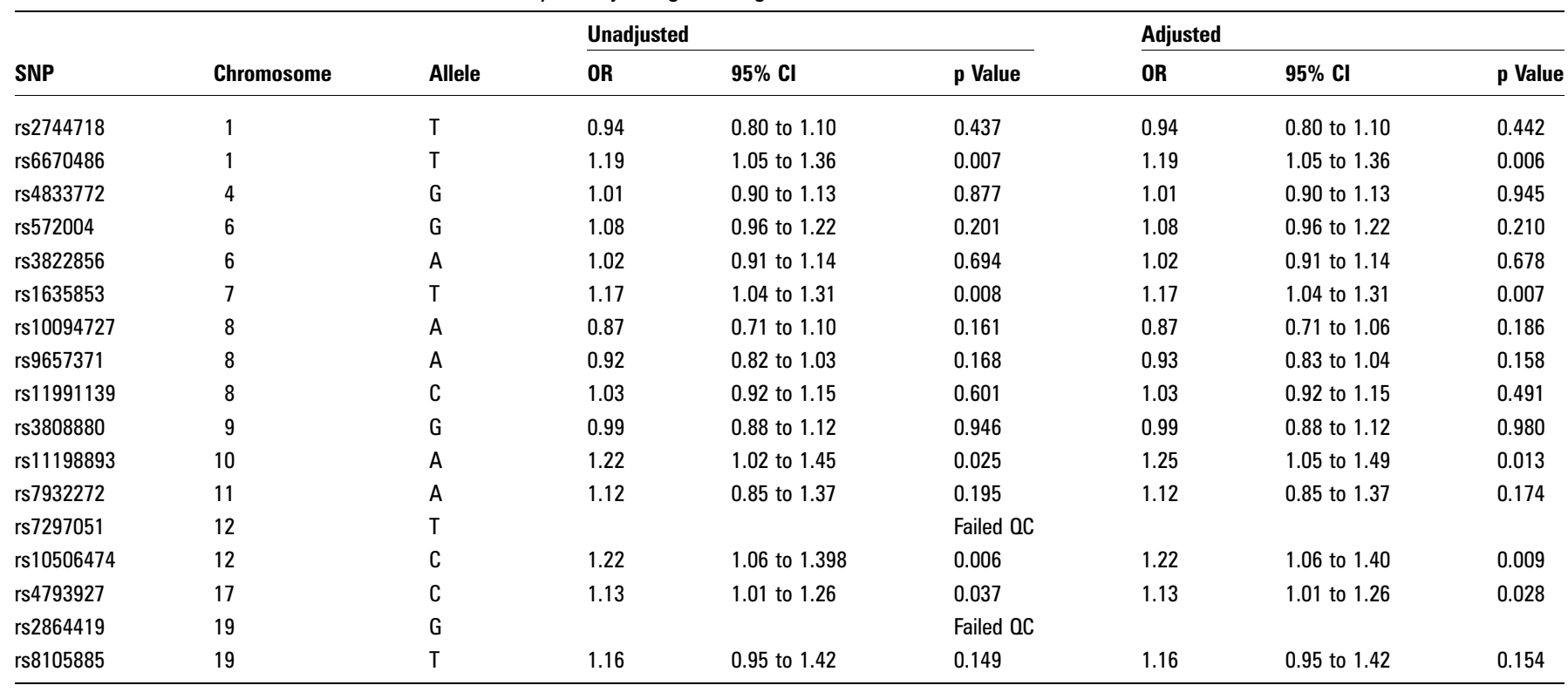

OC, qualtiy control; SNP, single nucleotide polymorphism. 
Table 8 Results of osteoarthritis association analysis adjusting for BMI

\begin{tabular}{|c|c|c|c|c|c|c|c|c|}
\hline \multirow[b]{2}{*}{ SNP } & \multirow[b]{2}{*}{ Chromosome } & \multirow[b]{2}{*}{ Allele } & \multicolumn{3}{|c|}{ Unadjusted } & \multicolumn{3}{|c|}{ Adjusted } \\
\hline & & & OR & $95 \% \mathrm{Cl}$ & p Value & OR & 95\% Cl & p Value \\
\hline rs4856346 & 3 & T & 1.06 & 0.94 to 1.19 & 0.380 & 1.06 & 0.94 to 1.19 & 0.614 \\
\hline rs7203219 & 16 & $\mathrm{~T}$ & 1.03 & 0.88 to 1.21 & 0.667 & 1.04 & 0.89 to 1.23 & 0.591 \\
\hline rs12149832 & 16 & $A$ & 1.12 & 1.01 to 1.24 & $2.9 \times 10^{-2}$ & 1.12 & 1.01 to 1.24 & 0.226 \\
\hline
\end{tabular}

BMI, body mass index; SNP, single nucleotide polymorphism.

\section{Author affiliations}

${ }^{1}$ Wellcome Trust Centre for Human Genetics, University of Oxford, Oxford, UK

${ }^{2}$ Nuffield Department of Orthopaedics, Rheumatology and Musculoskeletal Sciences,

University of Oxford, Oxford, UK

${ }^{3}$ Wellcome Trust Sanger Institute, Hinxton, Cambridge, UK

${ }^{4} \mathrm{NIHR}$ Biomedical Research Unit, University of Oxford, Oxford, UK

${ }^{5}$ MRC Epidemiology Resource Centre, University of Southampton, Southampton, UK

${ }^{6}$ Orthopaedic Department, Worcestershire Acute Hospitals NHS Trust, Worcester, UK

${ }^{7}$ Musculoskeletal Research Group, Newcastle University, Institute of Cellular

Medicine, The Medical School, Newcastle upon Tyne, UK

${ }^{8}$ Northumbria Healthcare NHS Foundation Trust, Wansbeck General Hospital,

Ashington, UK

${ }^{9}$ Academic Rheumatology, University of Nottingham, Nottingham, UK

${ }^{10}$ Institute of Cellular Medicine, Musculoskeletal Research Group, Newcastle

University, Newcastle upon Tyne, UK

${ }^{11}$ The Newcastle upon Tyne Hospitals NHS Trust Foundation Trust, The Freeman

Hospital, Newcastle upon Tyne, UK

${ }^{12}$ Centre for Integrated Genomic Medical Research, University of Manchester,

Manchester, UK

${ }^{13}$ Rheumatology Department, Worcestershire Acute Hospitals NHS Trust, Worcester, UK

${ }^{14}$ Institute of Genetics and Molecular Medicine, University of Edinburgh, Edinburgh, UK

${ }^{15}$ Department of Twin Research and Genetic Epidemiology, King's College London, London, UK

${ }^{16}$ Wellcome Trust Centre for Cell Matrix Research, University of Manchester,

Manchester, UK

${ }^{17}$ Academic Unit of Bone Metabolism, Department of Human Metabolism, University of Sheffield, Sheffield, UK

${ }^{18}$ Sheffield NIHR Bone Biomedical Research Unit, Centre for Biomedical Research,

Northern General Hospital, Sheffield, UK

Funding arCOGEN (http://www.arcogen.org.uk/) is funded by a special purpose grant from Arthritis Research UK (grant 18030). This study was funded by The Wellcome Trust (098051 and WT079557MA), Arthritis Research UK, The Wishbone Trust, The Collisson Foundation, The Botnar Foundation, The Lord Nuffield Orthopaedic Trust, The Jean Shanks foundation, NIHR Musculoskeletal Biomedical Research Unit and EU Fp7 Treat-OA. For Newcastle we acknowledge the support of the UK NIHR BRC for Ageing and Age-related disease award to the Newcastle upon Tyne Hospitals NHS Foundation Trust and the support of the Northumberland, Tyne and Wear CLRN. KC is a Botnar Research Fellow.

Contributors Data generation: KSE, KC, ADW, KP, LS, CML; GIANT: NKA, AC, PD, MD, JL, AM, WERO, SR, TDS, AMV, GAW, JMW, AR, NA, FB, MRR, IC; arCOGEN: EZ. Data analysis: KSE, KC, ADW, KP, EZ. Manuscript preparation: KSE, KC, KP, ADW, EZ. Critical reading and approval of manuscript: KSE, KC, ADW, KP, LS, CML, NKA, AC, PD, MD, JL, AM, WERO, SR, TDS, AMV, GAW, JMW, AR, NA, FB, MRR, IC, EZ. The first two authors contributed equally.

\section{Competing interests None.}

Ethics approval Ethics approval for this study was obtained from National Research Ethics Service (REC ref\# 07/H0606/150).

Provenance and peer review Not commissioned; externally peer reviewed.

Open Access This is an Open Access article distributed in accordance with the Creative Commons Attribution Non Commercial (CC BY-NC 3.0) license, which permits others to distribute, remix, adapt, build upon this work non-commercially, and license their derivative works on different terms, provided the original work is properly cited and the use is non-commercial. See: http://creativecommons.org/licenses/by-nc/3.0/

\section{REFERENCES}

1. Creamer P, Hochberg MC. Osteoarthritis. Lancet 1997;350:503-8.

2. Felson DT, Anderson JJ, Naimark A, et al. Obesity and knee osteoarthritis: the Framingham Study. Ann Intern Med 1988;109:18-24.
3. Felson DT, Zhang $Y$, Anthony JM, et al. Weight loss reduces the risk for symptomatic knee osteoarthritis in women: the Framingham study. Ann Intern Med 1992;116:535-9.

4. Apold H, Meyer HE, Espehaug B, et al. Weight gain and the risk of total hip replacement a population-based prospective cohort study of 265,725 individuals. Osteoarthritis Cartilage 2011;19:809-15.

5. Lohmander LS, Gerhardsson M, Rollof $\mathrm{J}$, et al. Incidence of severe knee and hip osteoarthritis in relation to different measures of body mass. A population-based prospective cohort study. Ann Rheum Dis 2008;68:490-6.

6. Goulston LM, Kiran A, Javaid MK, et al. Does obesity predict knee pain over fourteen years in women, independently of radiographic changes? Athritis Car Res 2011;63:1398-406.

7. Loos RJF, Lindgren CM, Li S, et al. Common variants near MC4R are associated with fat mass, weight and risk of obesity. Nat Genet 2008;40:768-75.

8. Frayling TM, Timpson NJ, Weedon MN, et al. A common variant in the FTO gene is associated with body mass index and predisposes to childhood and adult obesity. Science 2007;316:889-94.

9. Scuteri A, Sanna S, Chen WM, et al. Genome-wide association scans shows genetic variants in the FTO gene are associated with obesity-related traits. PLOS Genet 2007;3:e115.

10. Liu YJ, Liu XG, Wang L, et al. Genome-wide association scans identified CTNNBL1 as a novel gene for obesity. Hum Mol Gen 2008;17:1803-13.

11. Willer CJ, Speliotes EK, Loos RJ, et al. Six new loci associated with body mass index highlight a neuronal influence on body weight regulation. Nat Gen 2009; 41:25-34.

12. Thorleifsson G, Walters GB, Gudbjartsson DF, et al. Genome-wide association yields newq sequence variants at seven loci that associate with measures of obesity. Nat Gen 2009;41:18-24.

13. Speliotes E, Willer CJ, Berndt SI, et al. Association analyses of 249,796 individuals reveal 18 new loci associated with body mass index. Nat Gen 2010;42:937-48.

14. Spector TD, Reneland $\mathrm{RH}, \mathrm{Mah} \mathrm{S}$, et al. Association between a variation in LRCH1 and knee osteoarthritis: a genome-wide single-nucleotide polymorphism association study using DNA pooling. Arthritis Rheum 2006;54:524-32.

15. Lango Allen H, Estrada $\mathrm{K}$, Lettre $\mathrm{G}$, et al. Hundreds of variants clustered in genomic loci and biological pathways affect human height. Nature 2010;467:832-8.

16. Weedon MN, Lettre G, Freathy RM, et al. A common variant of HMGA2 is associated with adult and childhood height in the general population. Nat Gen 2007;39:1245-50.

17. Lettre $\mathbf{G}$, Jackson $A U$, Gieger $C$, et al. Identification of ten loci associated with height highlights new biological pathways in human growth. Nat Genet 2008;40:584-91.

18. Weedon MN, Lango $\mathrm{H}$, Lindgren $\mathrm{CM}$, et al. Genome-wide association analysis identifies 20 loci that influence height. Nat Genet 2008;40:575-83.

19. Sanna S, Jackson AU, Nagaraja R, et al. Common variants in the GDF5-UOCC region are associated with variation in human height. Nat Genet 2008;40: 198-203.

20. Miyamoto Y, Mabuchi A, Shi D, et al. Functional polymorphism in the $5^{\prime}$ UTR of GDF5 is associated with susceptibility to osteoarthritis. Nat Genet 2007;39:529-33.

21. Chapman K, Takahashi A, Meulenbelt I, et al. A meta-analysis of European and Asian cohorts reveals a global role of a functional SNP in the $5^{\prime}$ UTR of GDF5 with osteoarthritis susceptibility. Hum Mol Genet 2008;17:1497-504.

22. Evangelou $\mathbf{E}$, Chapman $\mathrm{K}$, Meulenbelt I, et al. Large-scale analysis of association between GDF5 and FRZB variants and osteoarthritis of the hip, knee, and hand. Arthritis Rheum 2009:60:1710-21.

23. Valdes AM, Evangelou E, Kerkhof HJ, et al. The GDF5 rs143383 polymorphism is associated with osteoarthritis of the knee with genome-wide statistical significance. Ann Rheum Dis 2011;70:873-5.

24. Valdes AM, McWilliams D, Arden NK, et al. Involvement of different risk factors in clinically severe large joint osteoarthritis according to the presence of hand interphalangeal nodes. Arthritis Rheum 2010;62:2688-95.

25. GIANT consortium. Membership of the GIANT consortium. http://hmg. oxfordjournals.org/content/suppl/2009/01/21/ddp041.DC1/ddp041 supp.pdf (accessed 1 May 2012). 
26. Consortium. Membership of the GIANT consortium. http://www.helmholtz-muenchen. de/epi/beitraege-zu-netzwerken/giant-genomewide-investigation-of-anthropometricmeasures/index.html (accessed 1 May 2012).

27. Panoutsopoulou K, Southam L, Elliott KS, et al. Insights into the genetic architecture of osteoarthritis from stage 1 of the arcOGEN study. Ann Rheum Dis. 2011;70:864-7.

28. Purcell S, Neale B, Todd-Brown K, et al. PLINK: a tool set for whole-genome association and population-based linkage analyses. Am J Hum Genet 2007:81:559-75.

29. Magi R, Morris AP. GWAMA: software for genome-wide association meta-analysis. BMC Bioinformatics 2010;11:288.

30. Marchini J, Howie B, Myers S, et al. A new multipoint method for genome-wide association studies by imputation of genotypes. Nat Genet 2007;39:906-13.

31. Estrada K, Styrkarsdottir U, Evangelou E, et al. Genome-wide meta-analysis identifies 56 bone mineral density loci and reveals 14 loci associated with risk of fracture. Nat Genet 2012;44:491-501.
32. Hart DJ, Spector TD. The relationship of obesity, fat distribution and osteoarthritis in women in the general population: the Chingford Study. J Rheumatol 1993;20:331-5.

33. $\mathbf{X u} \mathbf{L}$, Flahiff CM, Waldman BA, et al. Osteoarthritis-like changes and decreased mechanical function of articular cartilage in the joints of mice with the chondrodysplasia gene (cho). Arthritis Rheum 2003;48:2509-18.

34. Okano K, Tsukazaki T, Ohtsuru A, et al. Expression of parathyroid hormone-related peptide in human osteoarthritis. J Orthop Res 1997;15:175-80.

35. Tompson SW, Bacino CA, Safina NP, et al. Fibrochondrogenesis results from mutations in the COL11A1 type XI collagen gene. Am J Hum Genet 2010;87 708-12.

36. MacGregor AJ, Andrew T, Sambrook PN, et al. Structural, psychological, and genetic influences on low back and neck pain: a study of adult female twins. Arthritis Rheum 2004;51:160-7. 